\title{
Progressive ataxia with oculo-palatal tremor and optic atrophy.
}

Papachatzaki, MM; Ali, N; Arshad, Q; Cader, S; Peppas, I; Everett, C; Bronstein, AM;

Schmierer, K

(C) 2013 Springer-Verlag Berlin Heidelberg

Permission to reuse

For additional information about this publication click this link.

http://qmro.qmul.ac.uk/xmlui/handle/123456789/9611

Information about this research object was correct at the time of download; we occasionally make corrections to records, please therefore check the published record when citing. For more information contact scholarlycommunications@qmul.ac.uk 


\section{Journal of Neurology \\ Progressive ataxia with oculo-palatal tremor and optic atrophy. Asynch.CoverPage.ManuscriptDraft}

\begin{tabular}{|l|}
\hline Common.Text.ManuscriptNumber: \\
\hline Common.SubmissionDetails.FullTitle: \\
\hline Common.Labels.ArticleType \\
\hline $\begin{array}{l}\text { Common.SubmissionDetails.Correspondi } \\
\text { ngAuthor: }\end{array}$ \\
\hline
\end{tabular}

Common.SubmissionDetails.SecondaryC orrespondingAuthor:

Common.SubmissionDetails.Correspondi ngAuthorlnstitution:

\section{Common.SubmissionDetails.SecondaryC orrespondingAuthorInstitution:}

Common.SubmissionDetails.Correspondi ngAuthorEmail:

Common.SubmissionDetails.FirstAuthor: Common.SubmissionDetails.SecondaryFi rstAuthor:

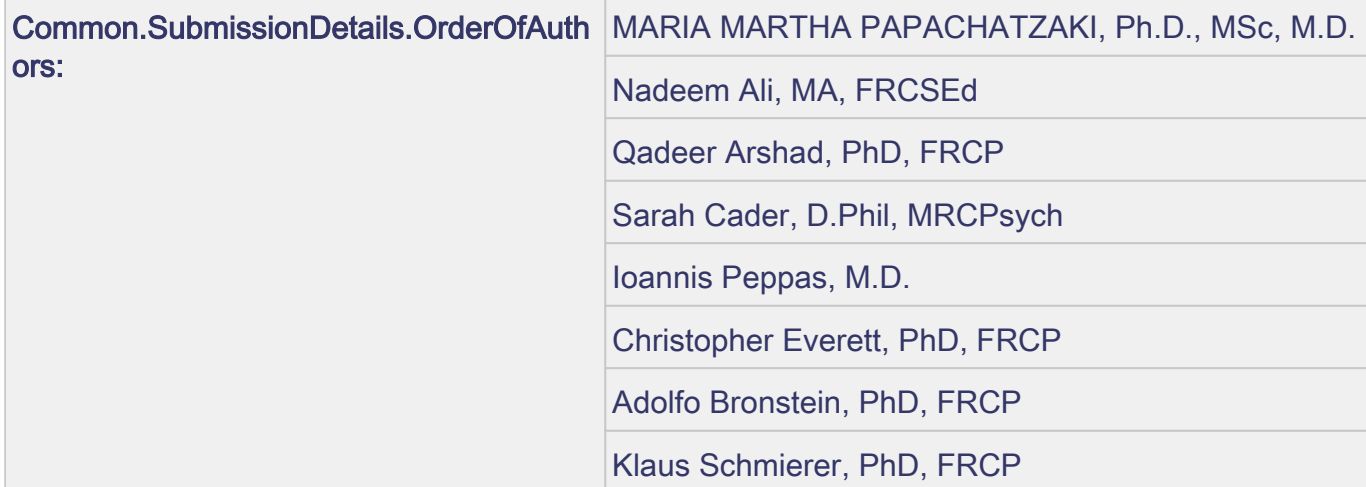

MARIA MARTHA PAPACHATZAKI, Ph.D., MSc, M.D.

Nadeem Ali, MA, FRCSEd

Qadeer Arshad, PhD, FRCP

Sarah Cader, D.Phil, MRCPsych

loannis Peppas, M.D.

Christopher Everett, PhD, FRCP

Adolfo Bronstein, PhD, FRCP

Klaus Schmierer, PhD, FRCP

Common.SubmissionDetails.Abstract:

Progressive ataxia palatal tremor (PAPT) is a rare neurological syndrome characterized by progressive cerebellar ataxia and palatal tremor. Sporadic as well as hereditary forms of PAPT have been described. Whereas sporadic PAPT is typically associated with bilateral pseudo-hypertrophy of the inferior olivary nuclei and progressive cerebellar atrophy, familial PAPT cases show brainstem and spinal cord atrophy in the absence of olivary signal changes on magnetic resonance imaging (MRI). Familial PAPT is often associated with upper motor neuron signs whereas sporadic PAPT cases are associated with cerebellar ataxia. Palatal tremor in the sporadic PAPT cases is characterised by non-periodic, low frequency oscillations (1-3 $\mathrm{Hz})$ of the posterior soft palate, often accompanied by branchial $(60 \%)$ and/or ocular $(30 \%)$ muscles oscillations.

We report a unique case of sporadic PAPT with bilateral optic atrophy and upper motor neuron signs.

Common.SubmissionDetails.ResponseTo Reviewers:
JOON-D-13-00941R1

Progressive ataxia with oculo-palatal tremor and optic atrophy.

Letter to the Editors

MARIA MARTHA PAPACHATZAKI, Ph.D., MSc, M.D.

London, Greater London UNITED KINGDOM

Blizard Institute, QMUL \& Barts and The London School of Medicine \& Dentistry
We thank both reviewers for favourably reviewing our manuscript.

Whilst reviewer \#1 felt the emphasis of the discussion should be slightly shifted away from the distinction between sporadic and familial PAPT towards "other neurological abnormalities associated with sporadic PAPT", reviewer \#2 emphasized the importance of highlighting the features normally associated with familial PAPT that 
were present in our case of sporadic PAPT.

In order to accommodate the valid views of both referees we left the bulk of the discussion untouched and inserted one sentence reminding the reader of the previously described association between sporadic PAPT and other neurodegenerative diseases (highlighted in yellow in the revised manuscript).

We hope this is satisfactory.

Common.SubmissionDetails.AuthorComm Dear Professor Filippi

ents:

Re: Progressive ataxia with oculopalatal tremor and optic atrophy

On behalf of the co-authors and myself I herewith submit the revised version of the abovementioned case report.

Thank you for considering our paper further for publication.

With kind regards,

Maria Papachatzaki 


\section{Re: JOON-D-13-00941R1 Response to reviewers}

We thank both reviewers for favourably reviewing our manuscript.

Whilst reviewer \#1 felt the emphasis of the discussion should be slightly shifted away from the distinction between sporadic and familial PAPT towards "other neurological abnormalities associated with sporadic PAPT", reviewer \#2 emphasized the importance of highlighting the features normally associated with familial PAPT that were present in our case of sporadic PAPT.

In order to accommodate the valid views of both referees we left the bulk of the discussion untouched and inserted one sentence reminding the reader of the previously described association between sporadic PAPT and other neurodegenerative diseases (highlighted in yellow in the revised manuscript).

We hope this is satisfactory. 


\title{
Progressive ataxia with oculo-palatal tremor and optic atrophy
}

\author{
Papachatzaki MM ${ }^{1,2}$, Ali $N^{3}$, Arshad $Q^{4,5}$, Cader $S^{6}$, Peppas $I^{1}$, Everett $C^{2}$, Bronstein $\mathrm{AM}^{4,5}$, \\ Schmierer $\mathrm{K}^{1,2}$. \\ ${ }^{1}$ Blizard Institute, Centre for Neuroscience, Barts and The London School of Medicine \& \\ Dentistry, London, UK \\ ${ }^{2}$ Royal London Hospital, Barts Health NHS Trust, Department of Neurology, London, UK \\ ${ }^{3}$ Moorfields Eye Hospital NHS Foundation Trust, London, UK \\ ${ }^{4}$ Imperial College London, Department of Medicine (Neuro-otology Unit), London, UK \\ ${ }^{5}$ Imperial College Healthcare NHS Trust, Department of Neurology, London, UK \\ ${ }^{6}$ Hampshire Hospitals NHS Foundation Trust, Basingstoke, UK
}

\section{Corresponding author}

Maria-Martha Papachatzaki, Blizard Institute, 4 Newark Street, London, E1 2AT, London, UK. m.papachatzaki@qmul.ac.uk, work tel: 02078828813, mobile: 07446055064.

$\begin{array}{ll}\text { Word count } & 604 \\ \text { Characters (title) } & 62 \\ \text { N references } & 10 \\ \text { N figures } & 1 \\ N \text { videos } & 2\end{array}$

Key words: cerebellar ataxia, oculopalatal tremor, optic atrophy, progressive ataxia and palatal tremor, myoclonus, movement disorders, neuro-ophthalmology.

Financial disclosures/conflict of interests related to article: None

Informed consent: Informed consent was obtained. 
JOON-D-13-00941R1 - Papachatzaki, et al. PAPT with optic atrophy 2

Dear Sirs,

A 42 year-old man of Polish extraction presented with a four year history of slowly deteriorating reading difficulties and gait imbalance. There was no evidence for alcohol or recreational substance abuse. Family history was unremarkable.

On examination he had tandem gait ataxia. Power was normal; no atrophies. Deep tendon reflexes were pronounced on the left with ipsilaterally extensor plantar response. He had upper limb past pointing, pronounced on the left. Visual acuity was $20 / 60$ on the right and $20 / 80$ on the left. Colour vision (Ishihara plates): $13 / 15$ on the right and 10/15 on the left. Humphrey 10:2 perimetry showed reduced sensitivity paracentrally in both eyes. Retinae appeared normal, however both optic discs showed temporal wedge-shaped pallor (Fig. 1). Examination of extra-ocular movements was supported by binocular 3D video oculography (Video 1). Pendular torsional nystagmus in primary gaze was detected, partly attenuated on left gaze. The nystagmus had two components, a baseline, low amplitude (1-2deg), symmetrical, pendular, torsional oscillation $(1.7-2 \mathrm{~Hz})$ and an irregular larger amplitude (2-5deg) nystagmus beating clockwise from the observer's point of view. Both nystagmus components were pronounced in the right eye. The torsional faster component included a large upwards component in the right eye ('left pendular hemi-seesaw nystagmus') [1] (Fig. 1). Non-periodic tremor (2Hz) of the soft palate and posterior pharyngeal wall muscles was evident (video 2).

Optical coherence Tomography of the peripapillary retinal nerve fibre layer confirmed bilateral segmental optic atrophy (Fig. 1). Full- field and multifocal electroretinograms were normal. Amplitudes of pattern reversal visual evoked potentials were bilaterally borderline reduced. 
JOON-D-13-00941R1 - Papachatzaki, et al. PAPT with optic atrophy 3

Haematology and biochemistry indices were normal as were vitamin $\mathrm{B}_{12}$, folic acid, thyroid function, syphilis and HIV 1/2 serology, and screening for onconeuronal and other auto-antibodies. Cerebrospinal fluid analysis was normal. Full GFAP gene sequencing failed to support presence of Alexander's disease [2]. Search for spinocerebellar ataxia expansions and/or duplications 1-3, 6, 7, 12 \& 20 was negative $[3,4]$ as were searches for mitochondrial mutations associated with (i) Leber's Hereditary Optic Atrophy [5], (ii) Mitochondrial Encephalomyopathy, Lactic acidosis and Stroke-like episodes, (iii) Myoclonic Epilepsy with Ragged Red Fibers, and (iv) Neuropathy, Ataxia and Retinitis Pigmentosa. Genetic testing for Optic Atrophy 1 [6] and Polymerase Gamma Gene mutations $[7,8]$ was also negative. MRI head and spinal cord showed hyper-intense signal in the medulla oblongata on $T_{2}$ weighted images suggesting bilateral pseudo-hypertrophy of inferior olivary nuclei, and mild cerebellar atrophy. MRI of optic nerves suggested reduced optic nerve diameter, pronounced on the left (Fig. 1).

To the best of our knowledge sporadic Progressive Ataxia Palatal Tremor (sPAPT) with bilateral optic atrophy and upper motor neuron (UMN) signs has never been reported. PAPT is a rare syndrome. SPAPT as well as familial (fPAPT) forms have been described. Whilst SPAPT is typically associated with bilateral pseudo-hypertrophy of the inferior olive and progressive cerebellar atrophy, people with fPAPT show brainstem and spinal cord atrophy in the absence of olivary changes. fPAPT is often associated with UMN signs whereas cases of sPAPT are associated with cerebellar ataxia [9]. Palatal tremor in sPAPT is characterised by non-periodic, low frequency oscillations $(1-3 \mathrm{~Hz})$ of the posterior soft palate, often accompanied by branchial (60\%) and/or ocular (30\%) muscles oscillations [10]. In our case palatal tremor was accompanied by oscillations of 
JOON-D-13-00941R1 - Papachatzaki, et al. PAPT with optic atrophy 4 branchial as well as ocular muscles to produce left 'pendular hemi-seeshaw' nystagmus.

Symptoms and signs were in line with lesions in the medulla oblongata, and an ocular degenerative abnormality, respectively [1]. An association between SPAPT and well described neurodegenerative diseases, for example, multiple system atrophy (MSA) has previously been reported [9]. However, early onset, fairly benign course, and lack of clinical and MRI findings characteristic of alternative neurodegenerative diseases render such association in our patient highly unlikely. Thus, in the absence of clinical, imaging or laboratory findings supporting the diagnosis of a known cause of PAPT, it is possible that our case represents a novel phenotype of sPAPT with bilateral optic atrophy.

Conflicts of interest All authors declare no conflicts of interest.

Ethical standard The authors declare they acted in accordance with the ethical standards laid down in the 1964 Declaration of Helsinki. 
JOON-D-13-00941R1 - Papachatzaki, et al. PAPT with optic atrophy 5

\section{References}

1. Lopez LI, Bronstein AM, Gresty MA, et al. (1996) Clinical and MRI correlates in 27 patients with acquired pendular nystagmus. Brain 119:465-472

2. Howard KL, Hall DA, Moon M, et al. (2008) Adult-onset Alexander disease with progressive ataxia and palatal tremor. Mov Disord 23:118-122

3. Storey E, Knight MA, Forrest SM, et al. (2005) Spinocerebellar ataxia type 20. Cerebellum 4: 55-57

4. Stricker S, Oberwahrenbrock T, Zimmermann H, et al. (2011) Temporal retinal nerve fibre loss in patients with spinocerebellar ataxia type 1. PLoS One 6: e23024

5. Morgia C, Achilli A, lommarini L, et al. (2008) Rare mtDNA variants in Leber hereditary optic neuropathy families with recurrence of myoclonus. Neurology 70:762-770.

6. Amati-Bonneau P, Valentino ML, Reynier P, et al. (2008) OPA1 mutations induce mitochondrial DNA instability and optic atrophy 'plus' phenotypes. Brain 131:338-351

7. Naviaux RK, Nguyen KV. (2005) POLG mutations associated with Alper's syndrome and mitochondrial DNA depletion. Ann Neurol 58: 491

8. Johansen KK, Bindoff LA, Rydland J, et al. (2008) Palatal tremor and facial dyskinesia in a patient with POLG1 mutation. Mov Disord 23:1624-1626

9. Samuel M, Torun N, Tuite PJ, et al. (2004) Progressive ataxia and palatal tremor (PAPT): clinical and MRI assessment with review of palatal tremors. Brain 127:1252-1268 10. Deuschl G, Toro C, Valls-Sole J, et al. (1994) Symptomatic and essential palatal tremor: Clinical, physiological and MRI analysis. Brain117:775-788 
JOON-D-13-00941R1 - Papachatzaki, et al. PAPT with optic atrophy 6

\section{Video 1}

3D video oculography of the right eye during fixation to a LED target, initially in primary gaze and then during fixation $15 \mathrm{deg}$ to the right, left and back to centre. Note the large, asymmetric, torsional, 'rotatory' nystagmus, which is larger and faster to the left (clockwise from the observer's point of view).

\section{Video 2}

Non periodic palatal tremor $(\sim 2 \mathrm{~Hz})$ with synchronous oscillations of the posterior pharyngeal wall muscles.

\section{Fig. 1}

Fundoscopy and ocular coherence tomography (OCT) ( $a=$ left eye; $b=$ right eye).

Bilateral pallor of the optic disc (arrows $a 1$ and b1). OCT of the retinal nerve fibre layer showing defect (summarized in pie charts) in all but the medial section of the left, and the inferior section of the right eye $(a 2 / a 3$ and $b 2 / b 3) . T_{2}$ weighted magnetic resonance imaging (MRI) showing bilateral pseudohypertrophy of the inferior olivary nuclei (arrow c, large arrow e), moderate cerebellar atrophy (small arrow d) and optic nerve atrophy, pronounced on the left (arrow d). 3D video oculography recordings ( $f$ ) showing pendular torsional nystagmus in primary gaze. The torsional component was associated with an apparent left and up beating torsional nystagmus (dashed arrows). A second smaller amplitude component of the pendular nystagmus was present (small arrow) with a frequency of $1.7-2 \mathrm{~Hz}$. 

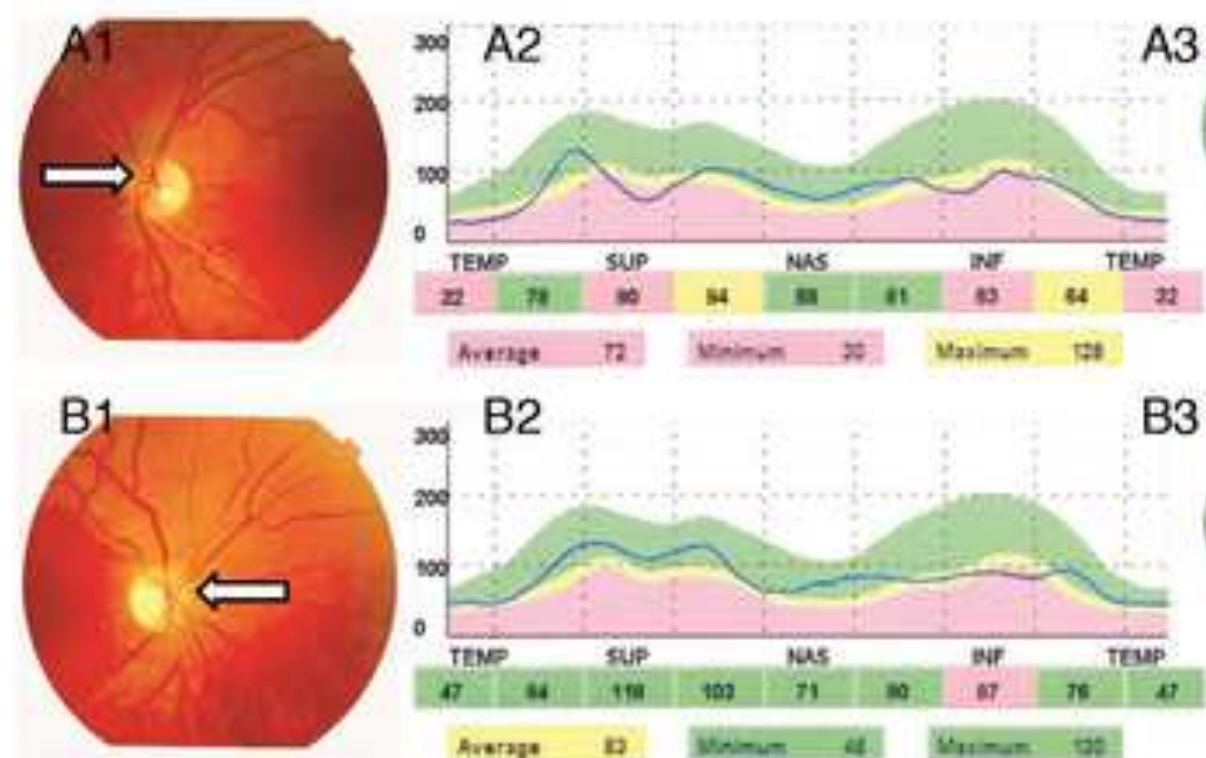

B2

B3
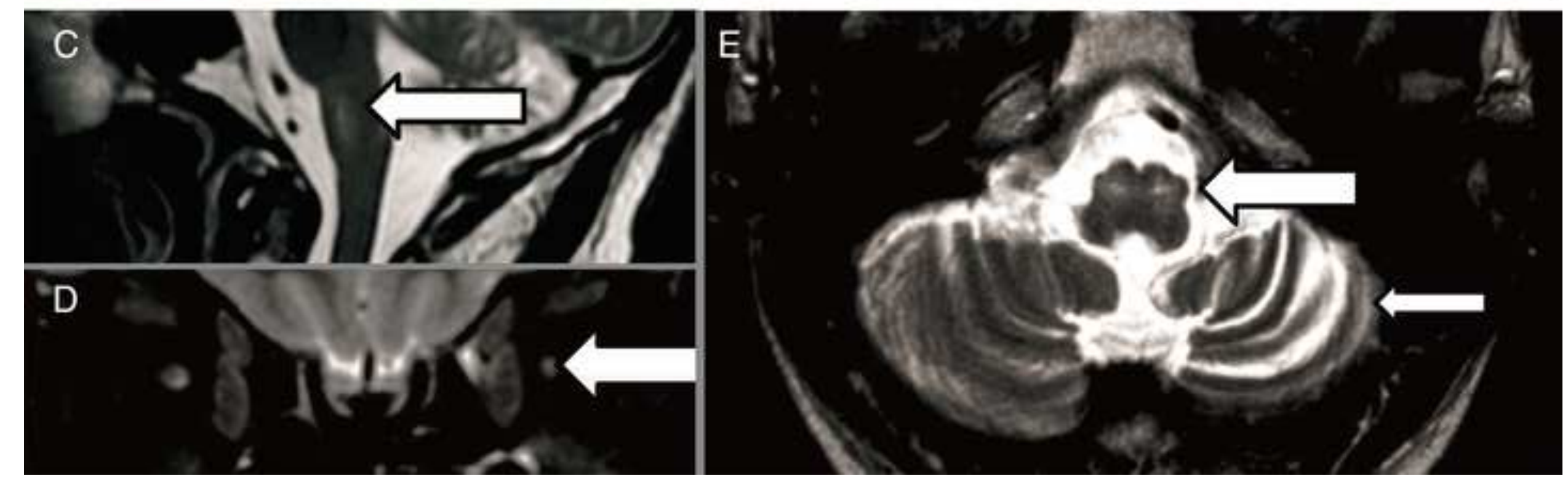
Click here to download Supplementary Material: Video 1 mpeg.m4v 
Click here to download Supplementary Material: Video 2 mpeg-1.m4v 